\title{
KINEMATIC AND DYNAMIC ANALYSIS OF NEW POLAR POSITIONING SYSTEM DEDICATED TO MECHATRONIC LASER GLASS ENGRAVING SYSTEM
}

\author{
R. TROCHIMCZUK \\ Department of Automatic Control and Robotics \\ Faculty of Mechanical Engineering \\ Bialystok University of Technology \\ ul. Wiejska 45C, 15-351 Białystok, POLAND \\ E-mail: r.trochimczuk@pb.edu.pl
}

\begin{abstract}
Analytical formulas describing the kinematics and dynamics of a multibody system of a new polar positioning system dedicated to mechatronic laser glass or other transparent dielectrics engraving system will be presented in this work. The analytical results will become in the later stages of the research the basis of numerical simulations. They will optimize the proposed solution of the positioning system.
\end{abstract}

Key words: polar positioning system, kinematic analysis, dynamic analysis, laser glass engraving.

\section{Introduction}

Since a man learned to produce glass from quartz sand many techniques have been developed in order to enhance the final form of the finished glass product. At first, in most cases the technical finishing of a glass product endured manual processing with the use of mechanical grinding. With the development of manufacturing techniques and the emergence of mass products, repetitive in terms of artistic form and the quality of execution of the final product, automatic CNC machines were introduced in the glass processing. This step greatly accelerated processing of the units. However engravings, cuts in the glass made using this type of machine was only made on the surface of the workpiece.

An important step that brought a new quality to the glass processing was the mechatronic laser engraving system which uses a laser as an instrument of machining (usually a pulsed laser). Those systems allowed engraving the subsurface of glass products. Typically, such engraving has the form of a threedimensional image created under the surface of the glass. This image is made of "point cloud" formed of microdefects (the size of one microdefect is approx. 200x200x400-600 micrometers), resulting from an exposure of the forming laser beam into the local area of the glass object.

This technique of decorating glass products as opposed to the classical mechanical treatment has its advantages, including: 1) the resulting image may take the form of a three-dimensional object, 2) the image is stable and is not damaged, e.g., due to the negative impact of external factors on the glass surface, mechanical scratches, dirt, etc., 3) engraved objects can take various spatial forms, 4) the images consist of a large number of points which gives the opportunity for a precise reproduction of the pattern created in the memory of the computer controlling the engraving process. A more detailed description of the laser engraving technology can be found in other works (Trochimczuk, 2009; Dostanko et al., 2002).

Modern technical implementations of laser engraving systems typically use the classical Cartesian positioning system for the positioning of the laser beam. The author of this work in his earlier publications (Trochimczuk, 2009; Trochimczuk and Gawrysiak, 2009; Trochimczuk, 2010; 2011) proposed a new solution, an alternative to the Cartesian positioning system - a polar positioning system. 
The design of this positioning system is patterned on the solution used in the structure of hard drives of personal computers.

In this paper the results of the analysis of the kinematics and dynamics of the positioning system are presented. Thanks to a simple design, high rigidity, lower price compared to the traditional Cartesian solutions of positioning systems and the use of mechatronic control, the polar positioning system can be used not only with the laser engraving glass or other transparent dielectrics systems, but also in other systems, e.g., using SLM technology (Selective Laser Melting) for the manufacture of dental implants and prosthetic or other biomedical applications. An example would be a laser correction of ophthalmic intraocular defects related to the human eye.

\section{Description of positioning system}

The present positioner consists of a rotary working arm and a rotary working table. Thanks to the mechatronic control system, coordinates of the point of structures are converted from the Cartesian coordinate system to the polar coordinate system, ipso facto this translates into a common rotary motion of these components. This allows proper focusing of the laser beam into the space of the glass workpiece. The layered engraving (from the lowermost layers toward the top of the object) allows the formation of points on a three-dimensional object. A detailed description of the construction of a new positioning system can be found in the works (Trochimczuk, 2009; 2010; 2011). Figure 1A contains the kinematic diagram of the positioner with the adopted coordinate systems for the analysis. It will be used to describe the system using Denavit-Hartenberg notation (D-H notation).

A)

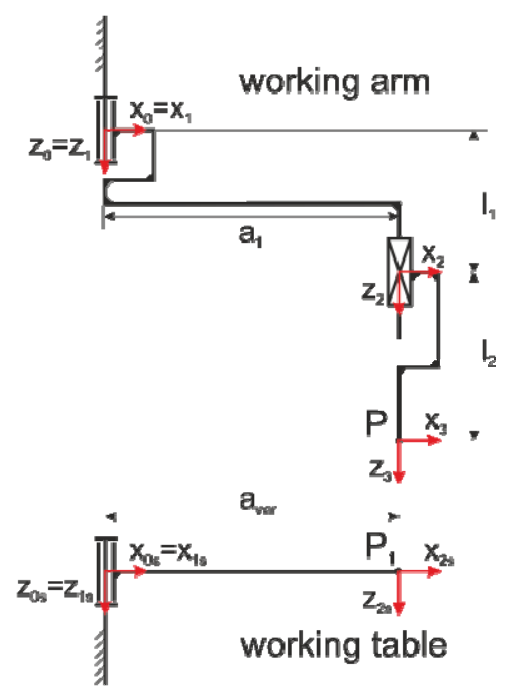

B)

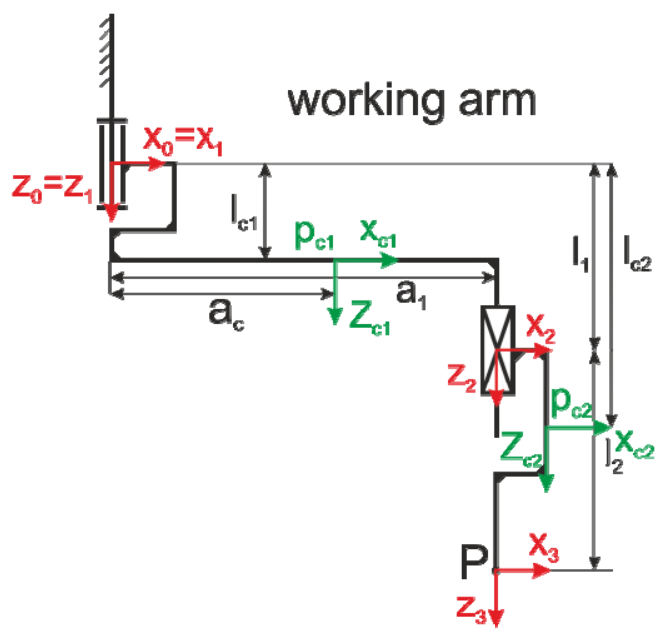

Fig.1. View of the mechanism of the polar positioning system (A) with coordinate system adopted to D-H notation, (B) working arm with the adopted coordinate system at the center of gravity.

For the purposes of a broader look at the positioning system from the point of view of the theory of machines and mechanisms, it can be assumed that the system of the working arm with the rotary working table of the polar positioning system can be treated as a closed kinematic chain of a four-bar linkage. The working arm length and the arm (the radius) of the working table are known - they are two arms of the fourbar linkage. As the third arm closing the kinematic chain, a laser beam can be assumed. The laser beam acts with no force in a specific area of the workpiece in the three-dimensional space. 


\section{Forward kinematics task of polar positioning system}

To describe the forward kinematics task the D-H notation given by Craig (1995) is used. The general form of the matrix is as follow

$$
{ }_{1}^{i-1} T=\left[\begin{array}{cccc}
\cos \theta_{i} & -\sin \theta_{i} & 0 & a_{i-1} \\
\sin \theta_{i} \cos \alpha_{i-1} & \cos \theta_{i} \cos \alpha_{i-1} & -\sin \alpha_{i-1} & -\sin \alpha_{i-1} l_{i} \\
\sin \theta_{i} \sin \alpha_{i-1} & \cos \theta_{i} \sin \alpha_{i-1} & \cos \alpha_{i-1} & \cos \alpha_{i-1} l_{i} \\
0 & 0 & 0 & 1
\end{array}\right] .
$$

D-H parameters adopted for the working arm and the rotary table of the polar positioning system are shown in Tab.1.

Table 1. D-H parameters of polar positioning system.

\begin{tabular}{|c|c|c|c|c|}
\hline \multicolumn{6}{|c|}{ Working arm - D-H parameters } \\
\hline $\boldsymbol{i}$ & $\boldsymbol{\alpha}_{\boldsymbol{i}-\boldsymbol{1}}$ & $\boldsymbol{a}_{\boldsymbol{i}-\boldsymbol{1}}$ & $\boldsymbol{\Theta}_{\boldsymbol{i}}$ & $\boldsymbol{l}_{\boldsymbol{i}}$ \\
\hline $0-1$ & 0 & 0 & $\Theta_{1}$ & 0 \\
\hline $1-2$ & 0 & $a_{1}$ & 0 & $l_{1}$ \\
\hline $2-3$ & 0 & 0 & 0 & $l_{2}$ \\
\hline \multicolumn{5}{|c|}{ Working Table - D-H parameters } \\
\hline $\boldsymbol{i}$ & $\boldsymbol{\alpha}_{\boldsymbol{i}-\boldsymbol{1}}$ & $\boldsymbol{a}_{\boldsymbol{i}-\boldsymbol{1}}$ & $\boldsymbol{\theta}_{\boldsymbol{i}}$ & $\boldsymbol{l}_{\boldsymbol{i}}$ \\
\hline $0-1$ & 0 & 0 & $\Theta_{2}$ & 0 \\
\hline $1-2$ & 0 & $a_{\text {var }}$ & 0 & 0 \\
\hline
\end{tabular}

The transformation matrix according to the accepted parameters for the working arm takes the form

$$
{ }_{p}^{0} T={ }_{1}^{0} T \cdot{ }_{2}^{1} T \cdot{ }_{3}^{2} T
$$

After substitution we obtain

$$
{ }_{p}^{0} T=\left[\begin{array}{cccc}
\cos \theta_{1} & -\sin \theta_{1} & 0 & a_{1} \cos \theta_{1} \\
\sin \theta_{1} & \cos \theta_{1} & 0 & a_{1} \sin \theta_{1} \\
0 & 0 & 1 & l_{1}+l_{2} \\
0 & 0 & 0 & 1
\end{array}\right]
$$

Similarly, for the rotary table we can write the transformation matrix

$$
{ }_{p s}^{0 s} T={ }_{1 s}^{0 s} T \cdot{ }_{2 s}^{1 s} T
$$

After substitution we obtain 


$$
{ }_{p s}^{o s} T=\left[\begin{array}{cccc}
\cos \theta_{2} & -\sin \theta_{2} & 0 & a_{\mathrm{var}} \cos \theta_{2} \\
\sin \theta_{2} & \cos \theta_{2} & 0 & a_{\mathrm{var}} \sin \theta_{2} \\
0 & 0 & 1 & 0 \\
0 & 0 & 0 & 1
\end{array}\right]
$$

For clarity of further proceedings only formulas for the working arm of the positioning system will be derived. Mathematical models for the rotary table can be obtained by analogy.

\section{Velocity and acceleration of the polar positioning system}

The polar positioning system consists of links. The motion of each link depends on the motions of the proceeding links. Therefore, you should determine the speed of individual joints in their frames of reference. Therefore the following mathematical formulas are taken

$$
\begin{aligned}
& { }^{i+1} \omega_{i+1}={ }_{i}^{i+1} R^{i} \omega_{i}+\dot{\theta}_{i+1}{ }^{i+1} \hat{Z}_{i+1}, \quad{ }^{i+1} v_{i+1}={ }_{i}^{i+1} R\left({ }^{i} v_{i}+{ }^{i} \omega_{i} \times{ }^{i} P_{i+1}\right)-\text { for rotational pair; } \\
& { }^{i+1} \omega_{i+1}={ }_{i}^{i+1} R^{i} \omega_{i}, \quad{ }^{i+1} v_{i+1}={ }_{i}^{i+1} R\left({ }^{i} v_{i}+{ }^{i} \omega_{i} \times{ }^{i} P_{i+1}\right)+\dot{d}_{i+1}{ }^{i+1} \hat{Z}_{i+1}-\text { for translation pair. }(4
\end{aligned}
$$

We obtain the matrix $R$ from the equation

$$
{ }_{i}^{i+1} R=\left({ }_{i}^{i+1} R\right)^{T}
$$

For the working arm we assume that velocities of the rotational pair 1 for $i=0$ equal

$$
{ }^{1} \omega_{1}=\left[\begin{array}{c}
0 \\
0 \\
\dot{\theta}_{1}
\end{array}\right], \quad{ }^{1} v_{l}=\left[\begin{array}{c}
0 \\
0 \\
0
\end{array}\right]
$$

Using formulas (4.1) and (4.2) we calculate the velocity of the rotary joint 2 for $i=1$

$$
{ }^{2} \omega_{2}={ }_{1}^{2} R^{1} \omega_{1}+\dot{\theta}_{2}{ }^{2} \hat{Z}_{2}=\left[\begin{array}{c}
0 \\
0 \\
\dot{\theta}_{1}+\dot{\theta}_{2}
\end{array}\right] ; \quad{ }^{2} v_{2}={ }_{1}^{2} R\left({ }^{1} v_{1}+{ }^{l} \omega_{1} \times{ }^{l} P_{2}\right)=\left[\begin{array}{c}
0 \\
a_{1} \dot{\theta}_{1} \\
0
\end{array}\right] .
$$

Similarly, for the translation joint 3 for $i=2$ we obtain

$$
{ }^{3} \omega_{3}={ }_{2}^{3} R^{2} \omega_{2}=\left[\begin{array}{c}
0 \\
0 \\
\dot{\theta}_{1}+\dot{\theta}_{2}
\end{array}\right] ; \quad{ }^{3} v_{3}={ }_{2}^{3} R\left({ }^{2} v_{2}+{ }^{2} \omega_{2} \times{ }^{2} P_{3}\right)+\dot{d}_{3}{ }^{3} \hat{Z}_{3}=\left[\begin{array}{c}
0 \\
a_{1} \dot{\theta}_{1} \\
\dot{d}_{3}
\end{array}\right] .
$$




\section{Forces and torques of the positioning system}

The working arm is loaded with a static force. Thus we calculate the forces and torques, respectively, using Eqs (5.1) and (5.2)

$$
\begin{aligned}
& { }^{i} f_{i}={ }_{i+1}^{i} R^{i+1} f_{i+1}, \\
& { }^{i} n_{i}={ }_{i+1}^{i} R^{i+1} n_{i+1}+{ }^{i} P_{i+1} \times{ }^{i} f_{i} .
\end{aligned}
$$

Forces and torques acting on the working arm in their coordinate systems are as follows

$$
{ }^{3} f_{3}=\left[\begin{array}{l}
f_{x} \\
f_{y} \\
f_{z}
\end{array}\right], \quad{ }^{3} n_{3}=\left[\begin{array}{c}
m_{x} \\
m_{y} \\
m_{z}
\end{array}\right] .
$$

We obtain, respectively

$$
\begin{aligned}
& { }^{2} f_{2}={ }_{3}^{2} R^{3} f_{3}=\left[\begin{array}{l}
f_{x} \\
f_{y} \\
f_{z}
\end{array}\right], \quad{ }^{2} n_{2}={ }_{3}^{2} R^{3} n_{3}+{ }^{2} P_{3} \times{ }^{2} f_{2}=\left[\begin{array}{c}
m_{x}-l_{2} f_{y} \\
m_{y}+l_{2} f_{x} \\
0
\end{array}\right] . \\
& { }^{1} f_{1}={ }_{2}^{1} R^{2} f_{2}=\left[\begin{array}{l}
f_{x} \\
f_{y} \\
f_{z}
\end{array}\right], \quad{ }^{1} n_{1}={ }_{2}^{1} R^{2} n_{2}+{ }^{1} P_{2} \times{ }^{1} f_{1}=\left[\begin{array}{c}
m_{x}-l_{2} f_{y}-l_{1} f_{y} \\
m_{y}+l_{2} f_{x}+l_{1} f_{x}-a_{1} f_{z} \\
a_{1} f_{y}
\end{array}\right] .
\end{aligned}
$$

The forces and the torques of drives $\tau_{i}$ of the polar positioning system may be calculated respectively for the rotary pair (5.6) and the translation pair (5.7)

$$
\begin{gathered}
\tau_{i}={ }^{i} n_{i}^{T} \cdot{ }^{i} \hat{Z}_{i}, \\
\tau_{i}={ }^{i} f_{i}^{T} \cdot{ }^{i} \hat{Z}_{i} .
\end{gathered}
$$

After substitution we obtain

$$
\begin{aligned}
& \tau_{1}={ }^{l} n_{1}{ }^{T} \cdot{ }^{1} \hat{Z}_{1}=a_{1} f_{y}, \\
& \tau_{2}={ }^{2} f_{2}^{T} \cdot{ }^{2} \hat{Z}_{2}=f_{z} .
\end{aligned}
$$




\section{Jacobians}

Velocity of the arm of the polar positioning system can be written as

$$
{ }^{3} v_{3}={ }^{3} J_{v_{3}} \cdot \dot{q}=\left[\begin{array}{cc}
0 & 0 \\
a_{1} & 0 \\
0 & 1
\end{array}\right]\left[\begin{array}{l}
\dot{\theta}_{1} \\
\dot{d}_{3}
\end{array}\right]
$$

where ${ }^{3} J_{v_{3}}$ is Jacobian of velocity, whereas the force and torques can be described by the following equation

$$
\tau=J_{F} \cdot f=\left[\begin{array}{ccc}
0 & a_{1} & 0 \\
0 & 0 & 1
\end{array}\right]\left[\begin{array}{l}
f_{x} \\
f_{y} \\
f_{z}
\end{array}\right]
$$

where $J_{F}$ is the Jacobian of static forces.

Considering the relationship between the Jacobian of velocity and Jacobian of static forces you can write that $\left(J_{F}\right)^{T}=J_{v}$. Comparing the results, we can conclude that the calculations were performed correctly.

\section{Dynamics of the polar positioning system}

To describe the dynamics of the working arm Lagrangian formalism will be used, allowing the analysis of the system, taking energy conversion into account. The equation of motion in a general form is

$$
\tau=M(q) \ddot{q}+V(q, \dot{q})+G(q)
$$

where: $M(q)$ - matrix of inertia (mass matrix) of the manipulator; $V(q, \dot{q})$ - vector of the centrifugal and Coriolis terms; $G(q)$ - vector of gravity terms.

The mathematical formula for calculating the inertia matrix of the general form can be written as

$$
M(q)=\sum_{i=1}^{n} m_{i} J_{v_{i}}{ }^{T} J_{v_{i}}+\sum_{i=1}^{n} J_{\omega_{i}}{ }^{T} I_{c_{i}} J_{\omega_{i}}
$$

where $m_{i}$ - mass of each joint; $I_{c_{i}}$ - tensors of inertia of joints relative to the centers of gravity; $p_{c_{i}}-$ position of the center of gravity of the joint $i$ relative to the coordinate system 0 -initial.

For that case it can be written

$$
J_{v_{i}}=\left[\begin{array}{llllllll}
\frac{\partial p_{c_{i}}}{\partial q_{1}} & \frac{\partial p_{c_{i}}}{\partial q_{2}} & \ldots & \frac{\partial p_{c_{i}}}{\partial q_{i}} & 0 & 0 & \ldots & 0
\end{array}\right]
$$




$$
J_{\omega_{i}}=\left[\begin{array}{llllllll}
\bar{\eta}_{1}\left({ }_{1}^{i} R^{l} \hat{Z}_{1}\right) & \bar{\eta}_{2}\left({ }_{2}^{i} R^{2} \hat{Z}_{2}\right) & \ldots & \bar{\eta}_{i}\left({ }_{i}{ }^{i} R^{i} \hat{Z}_{i}\right) & 0 & 0 & \ldots & 0
\end{array}\right] .
$$

In order to determine $p_{c_{i}}$ homogeneous operators ${ }_{p_{c_{i}}}^{0} T$ should be determined as presented in Fig.1B. For the adopted coordinate system we can write

$$
\begin{aligned}
& { }_{p_{c_{l}}}^{0} T={ }_{l}^{0} T_{p_{c_{l}}}^{l} T=\left[\begin{array}{cccc}
\cos \theta_{l} & -\sin \theta_{l} & 0 & a_{c} \cos \theta_{l} \\
\sin \theta_{l} & \cos \theta_{l} & 0 & a_{c} \sin \theta_{l} \\
0 & 0 & 1 & l_{c_{l}} \\
0 & 0 & 0 & 1
\end{array}\right], \quad p_{c_{l}}=\left[\begin{array}{c}
a_{c} \cos \theta_{l} \\
a_{c} \sin \theta_{l} \\
l_{c_{l}}
\end{array}\right] ; \\
& { }_{p_{c 2}}^{0} T={ }_{2}^{0} T_{p_{c 2}}^{2} T=\left[\begin{array}{cccc}
\cos \theta_{1} & -\sin \theta_{1} & 0 & 2 a_{1} \cos \theta_{1} \\
\sin \theta_{1} & \cos \theta_{1} & 0 & 2 a_{1} \sin \theta_{1} \\
0 & 0 & 1 & l_{1}+l_{c_{2}} \\
0 & 0 & 0 & 1
\end{array}\right], \quad p_{c_{2}}=\left[\begin{array}{c}
2 a_{1} \cos \theta_{1} \\
2 a_{1} \sin \theta_{1} \\
l_{1}+l_{c_{2}}
\end{array}\right] .
\end{aligned}
$$

Having calculated $p_{c_{i}}$ we calculate the Jacobian of linear velocity which takes the form of

$$
J_{v_{1}}=\left[\begin{array}{ll}
\frac{\partial p_{c_{1}}}{\partial \theta_{1}} & 0
\end{array}\right]=\left[\begin{array}{cc}
-a_{c} \sin \theta_{1} & 0 \\
a_{c} \cos \theta_{1} & 0 \\
0 & 0
\end{array}\right], \quad J_{v_{2}}=\left[\begin{array}{ll}
\frac{\partial p_{c_{2}}}{\partial \theta_{1}} & \frac{\partial p_{c_{2}}}{\partial l_{1}}
\end{array}\right]=\left[\begin{array}{cc}
-2 a_{1} \sin \theta_{1} & 0 \\
2 a_{1} \cos \theta_{1} & 0 \\
0 & 1
\end{array}\right] .
$$

Having calculated the Jacobians we calculate the contribution of inertia of joints to the mass matrix

$$
m_{l} J_{v_{l}}{ }^{T} J_{v_{l}}=\left[\begin{array}{cc}
a_{c}{ }^{2} m_{1}\left(\cos \theta_{l}+\sin \theta_{1}\right) & 0 \\
0 & 0
\end{array}\right], \quad m_{2} J_{v_{2}}{ }^{T} J_{v_{2}}=\left[\begin{array}{cc}
4 a_{1}{ }^{2} m_{2} & 0 \\
0 & m_{2}
\end{array}\right] .
$$

The Jacobian of angular velocity can be calculated by using Eqs (7.9), assuming that $\bar{\eta}_{l}=1$ - for rotation pair, $\bar{\eta}_{2}=0-$ for translation pair

$$
J_{\omega_{1}}=\left[\bar{\eta}_{1}\left(\begin{array}{ll}
\left.{ }_{1}^{1} R^{1} \hat{Z}_{1}\right) & 0
\end{array}\right]=\left[\begin{array}{ll}
0 & 0 \\
0 & 0 \\
1 & 0
\end{array}\right], \quad J_{\omega_{2}}=\left[\bar{\eta}_{1}\left({ }_{1}^{2} R^{1} \hat{Z}_{1}\right) \quad \bar{\eta}_{2}\left({ }_{2}^{2} R^{2} \hat{Z}_{2}\right)\right]=\left[\begin{array}{ll}
0 & 0 \\
0 & 0 \\
1 & 0
\end{array}\right] .\right.
$$

For modeling it is assumed that the inertia tensors are diagonal

$$
I_{c_{2}}=\left[\begin{array}{ccc}
I_{x x_{2}} & 0 & 0 \\
0 & I_{y y_{2}} & 0 \\
0 & 0 & I_{z z_{2}}
\end{array}\right], \quad I_{c_{1}}=\left[\begin{array}{ccc}
I_{x x_{1}} & I_{x y_{1}} & I_{x z_{1}} \\
I_{y x_{1}} & I_{y y_{1}} & I_{y z_{1}} \\
I_{z x_{1}} & I_{z y_{1}} & I_{z z_{1}}
\end{array}\right] .
$$
matrix.

Components resulting from the rotation reflecting the contribution of inertia of joints to the inertia 


$$
J_{\omega_{1}}{ }^{T} I_{c_{1}} J_{\omega_{1}}=\left[\begin{array}{cc}
I_{z z_{1}} & 0 \\
0 & 0
\end{array}\right], \quad J_{\omega_{2}}{ }^{T} I_{c_{2}} J_{\omega_{2}}=\left[\begin{array}{cc}
I_{z z_{2}} & 0 \\
0 & 0
\end{array}\right] .
$$

The inertia matrix can be written in a general form

$$
M=\left[\begin{array}{cc}
4 a_{1}{ }^{2} m_{2}+a_{c}{ }^{2} m_{l}\left(\cos \theta_{1}+\sin \theta_{l}\right)+I_{z z_{l}}+I_{z z_{2}} & 0 \\
0 & m_{2}
\end{array}\right] .
$$

The kinetic energy of the system can be written in a general form

$$
K=\frac{1}{2} \dot{q}^{T} M(q) \dot{q}
$$

Inserting Eqs (7.13) into (7.1) we obtain

$$
\frac{d}{d t}\left(\frac{\partial K}{\partial \dot{q}}\right)-\frac{\partial K}{\partial q}=M \ddot{q}+\dot{M} \dot{q}-\frac{1}{2}\left[\begin{array}{c}
\dot{q}^{T} \frac{\partial M}{\partial q_{1}} \dot{q} \\
\dot{q}^{T} \frac{\partial M}{\partial q_{2}} \dot{q} \\
\cdots \\
\cdots \\
\dot{q}^{T} \frac{\partial M}{\partial q_{n}} \dot{q}
\end{array}\right] .
$$

The matrix of the centrifugal and Coriolis forces $V(q, \dot{q})$ for the working arm of the polar positioning system can be written as

$$
V(q, \dot{q})=\dot{M} \dot{q}-\frac{1}{2}\left[\begin{array}{c}
\dot{q}^{T} \frac{\partial M}{\partial q_{1}} \dot{q} \\
\dot{q}^{T} \frac{\partial M}{\partial q_{2}} \dot{q}
\end{array}\right]=\left[\begin{array}{ll}
\dot{m}_{11} & \dot{m}_{12} \\
\dot{m}_{21} & \dot{m}_{22}
\end{array}\right] \dot{q}-\frac{1}{2}\left[\begin{array}{c}
\dot{q}^{T}\left(\begin{array}{ll}
m_{111} & m_{121} \\
m_{121} & m_{221}
\end{array}\right) \dot{q} \\
\dot{q}^{T}\left(\begin{array}{ll}
m_{112} & m_{122} \\
m_{122} & m_{222}
\end{array}\right) \dot{q}
\end{array}\right]
$$

where $\quad m_{111}=\frac{\partial m_{11}}{\partial q_{1}}, \quad m_{222}=\frac{\partial m_{22}}{\partial q_{2}}, \quad \dot{m}_{11}=m_{111} \dot{q}_{1}+m_{112} \dot{q}_{2}$.

According to Christoffel's symbols it can be written

$$
b_{i j k}=\frac{1}{2}\left(m_{i j k}+m_{i k j}-m_{j k i}\right), \quad m_{i j k}=\frac{\partial m_{i j}}{\partial q_{k}},
$$




$$
\begin{aligned}
& V(q, \dot{q})=\left[\begin{array}{ll}
\frac{1}{2}\left(m_{111}+m_{111}-m_{111}\right) & \frac{1}{2}\left(m_{122}+m_{122}-m_{221}\right) \\
\frac{1}{2}\left(m_{211}+m_{211}-m_{112}\right) & \frac{1}{2}\left(m_{222}+m_{222}-m_{222}\right)
\end{array}\right]\left[\begin{array}{c}
\dot{q}_{1}^{2} \\
\dot{q}_{2}^{2}
\end{array}\right]+ \\
& +\left[\begin{array}{l}
m_{112}+m_{121}-m_{121} \\
m_{212}+m_{221}-m_{122}
\end{array}\right]\left[\begin{array}{ll}
\dot{q}_{1} & \dot{q}_{2}
\end{array}\right] .
\end{aligned}
$$

You can also write for the working arm that

$$
V=C(q)\left[\begin{array}{c}
\dot{q}_{1}^{2} \\
\dot{q}_{2}^{2}
\end{array}\right]+B(q)\left[\dot{q}_{1} \dot{q}_{2}\right]
$$

where $C(q)=\left[\begin{array}{ll}b_{111} & b_{122} \\ b_{211} & b_{222}\end{array}\right]-$ Coriolis forces, $B(q)=\left[\begin{array}{l}2 b_{112} \\ 2 b_{212}\end{array}\right]-$ centrifugal force.

Hence

$$
V(q, \dot{q})=\left[\begin{array}{cc}
\frac{1}{2}\left[a_{c}{ }^{2} m_{l}\left(-\sin \theta_{l}+\cos \theta_{l}\right)\right] & 0 \\
0 & 0
\end{array}\right]\left[\begin{array}{c}
\dot{\theta}_{l}^{2} \\
i_{l}^{2}
\end{array}\right]+\left[\begin{array}{l}
0 \\
0
\end{array}\right]\left[\begin{array}{ll}
\dot{\theta}_{l} & i_{l}
\end{array}\right] .
$$

In the following part of the calculation the force of gravity should be taken into account. The energy of the working arm of the polar positioning system $U$ is the sum of the potential energy of its joints.

$$
U=\sum_{i=1}^{n} U_{i}
$$

The potential energy of joint $i$

$$
U_{i}=m_{i} g h_{i}+U_{0}
$$

where: $g$ - acceleration due to gravity, $U_{0}$ - the potential energy of the reference.

The general form can be written

$$
U_{i}=m_{i}\left(-g^{T} p_{c_{i}}\right)+U_{0}
$$

The value of the attraction vector can be written as

$$
g=\left[\begin{array}{c}
0 \\
0 \\
-g
\end{array}\right]
$$

The gravity force is a derivative of the potential energy, hence 


$$
G_{j}=\frac{\partial U}{\partial q_{j}}=-\sum_{i=1}^{n}\left(m_{i} g^{T} \frac{\partial p_{c_{i}}}{\partial q_{j}}\right) .
$$

Taking into account the previous mathematical formulas, the force of gravity for the working arm of the polar positioning system can be written as

$$
G=-\left(J_{v_{I}}{ }^{T} m_{1} g+J_{v_{2}}^{T} m_{2} g\right)=\left[\begin{array}{c}
0 \\
g m_{2}
\end{array}\right] .
$$

In the presented dynamic model of the polar positioning system friction was omitted. However, it should be considered in the dynamic equation, assuming at least an additional effect of Coulomb and viscous friction. In the assumed dynamic model the stiffness factor of the joints of the positioning system is also omitted, i.e., bending effects, which may cause additional resonances of system.

\section{Conclusions}

The analytical solutions to the forward kinematics task and dynamics equation describing the polar positioning system will be used in further stages of research to verify the result obtained from the numerical study of solid models created in the environment of SolidWorks program. The research on the polar positioning system from the point of view of the simplicity of its implementation, lower prices (compared to traditional Cartesian positioners), high stiffness, high velocity and acceleration of displacement and possibility the implementation of solution not only in laser engraving system, but also earlier mentioned in the introduction biomedical applications are justified.

This research has been done as a part of a project No. S/WM/1/2012, which is funded by Bialystok's Technical University, Poland.

\section{Nomenclature}

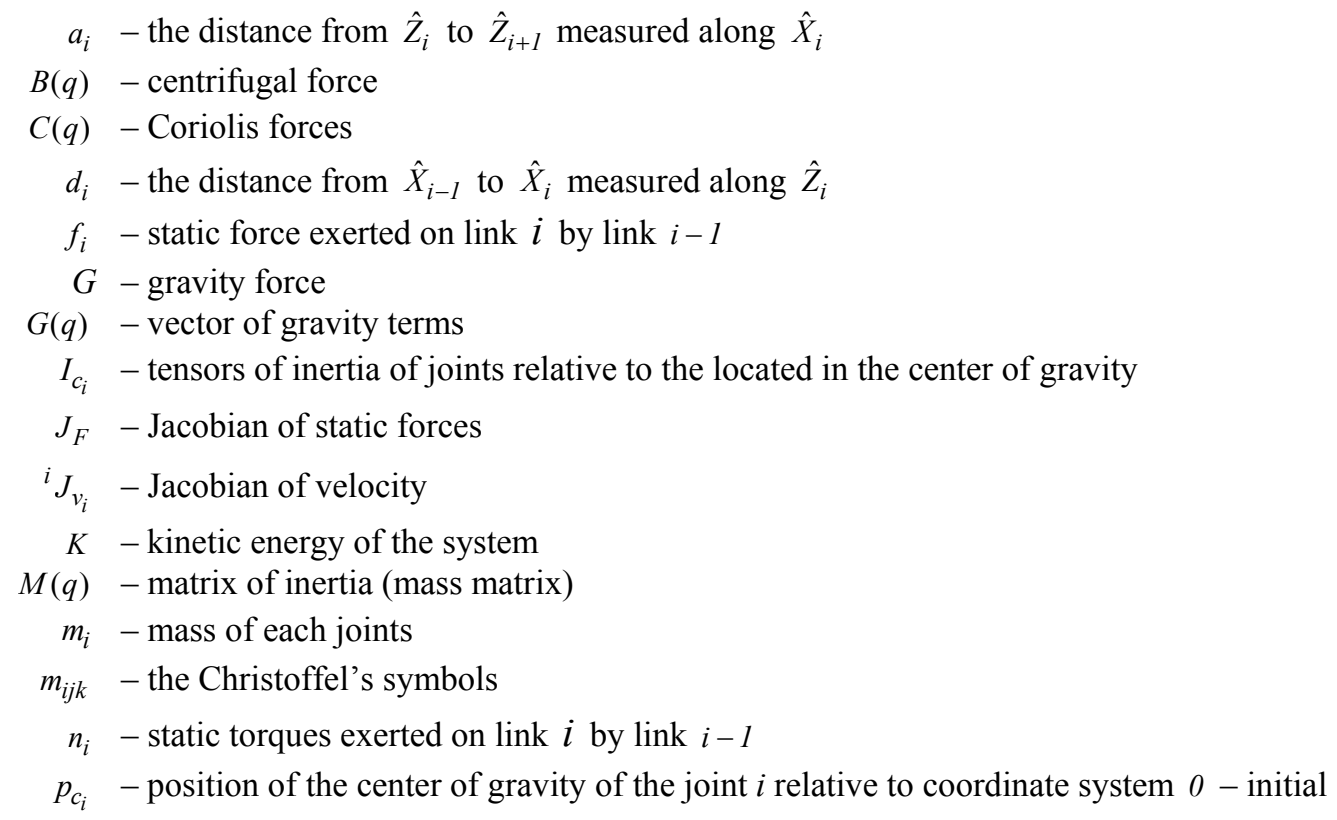


$R$ - rotation matrix

${ }_{1}^{i-l} T$ - transformation matrix 1 coordinate system relative to $i-1$ coordinate system

$U$ - potential energy

$V(q, \dot{q})$ - vector of the centrifugal and Coriolis terms

$\alpha_{i}$ - the angle from $\hat{Z}_{i}$ to $\hat{Z}_{i+1}$ measured about $\hat{X}_{i}$

$\theta_{i} \quad$ - the angle from $\hat{X}_{i-1}$ to $\hat{X}_{i}$ measured about $\hat{Z}_{i}$

${ }^{i+1} v_{i+1}-$ the linear velocity of the origin of frame $\{i+1\}$ is the same as that of the origin of frame $\{i\}$ plus a new component caused by rotational velocity of link $i$

$\tau-$ generalized forces are in individual joints

$\tau_{i}-$ joint torque required to maintain the static equilibrium

${ }^{i+1} \omega_{i+1}-$ description of the angular velocity of link $i+l$ with respect to frame $\{i+l\}$

\section{References}

Craig J.J. (1995): Introduction to Robotics. Mechanics and control. - Warsaw: WNT.

Dostanko A.P., Tolochko N.K. and Karpovich S.E. (2002): Technology and technique of precise laser modification of solid-state structures (in Russian). - Mińsk, Białoruś: Technoprint.

Trochimczuk R. (2009): Mechatronic positioning of beam of impulse laser in device for forming three-dimensional object inside glass (in Polish). - Ph. D. thesis. AGH, Cracow.

Trochimczuk R. (2010): Study of construction a new polar positioning system for laser device for 3D subsurface glass engraving (in Polish). - Acta Mechanica et Automatica, vol.4, No.1, pp.96-100, Białystok.

Trochimczuk R. (2011): Study of design of the mechanical actuator system of polar positioning system of laser engraving device. - In: Mechatronic design. Issues selected (in Polish), AGH, Cracow, pp.193-201.

Trochimczuk R. and Gawrysiak M. (2009): Analysis of polar positioning system in laser device for glass engraving. Solid State Phenomena, vols 147-149, Trans Tech Publications, Switzerland, Zurich, pp.107-112.

Received: June 3, 2014

Revised: October 1, 2014 